\title{
Research on Dynamic Equivalent for Hydropower Generator Group Based on Phasor Measurement Information Part II: Application in Real Power System Huabo SHI ${ }^{1, a}$, Lijie DING ${ }^{1, b}$, Hua ZHANG ${ }^{1, c}$, Gang CHEN ${ }^{4, d}$ \\ ${ }^{1}$ State grid Sichuan Electric Power Research Institute, Chengdu, 610072, China aemail:shbo87@163.com, bemail:ding_lijie@163.com, cemail:zhanghua002@163.com demail:gangchen08@gmail.com
}

Keywords: PMU; Dynamic equivalent; Disturbance Data Selection; Application

\begin{abstract}
In order to make the dynamic equivalent method for hydropower generator group based on phasor measurement information more suitable for the actual grid. A index use tie-line power oscillation to evaluate disturbance intensity is presented .Based on this index, consider disturbance location and it's distance to the equivalent area, a disturbance selection method is proposed. Based on the equivalent method, dynamic equivalent online identification system is developed. The disturbance selection method proposed is verified using PMU measured disturbances data of Sichuan power grid China.
\end{abstract}

\section{Introduction}

Along with the increase of phasor measurement device(PMU) in the real power system, dynamic equivalent for hydropower generator group based on phasor measurement information has a broad application prospect.

Dynamic equivalence is one of the most traditional research direction of power system, a large amount of research on the system dynamic equivalent model and parameter identification method are studied by many scholars and achieved abundant research results. But there is a little research on the application problem of equivalent in real power system .

Reference 1-2 used 39 bus system with 10 generators to verify equivalent method the paper proposed; reference 3 did research on the equivalent method application in Tianjin power grid China, but disturbance selection is not involved. Existing literatures verify their equivalent method in simulation software generally. There is little Reference studies the application of equivalent method and disturbance selection in actual power grid, which affect the application of the equivalent method and the accuracy of equivalent.

Therefore, this paper researches on dynamic equivalent for hydropower generator group based on phasor measurement information of tie-line. A index use tie-line power oscillation to evaluate disturbance intensity is presented, based on this index, consider disturbance location and it's distance to the equivalent area, a disturbance selection method is proposed. And dynamic equivalent modeling software is developed.

The equivalent method and disturbance selection method proposed is verified using PMU measured disturbances data of Sichuan power grid. Example analysis shows that disturbance data selection and dynamic equivalent method are effective and feasible. The method can make hydropower generator group dynamic characteristics simulation more accurate.

\section{Review of the dynamic equivalent method}

According to reference 4, the basic dynamic equivalent method for hydropower generator group based on phasor measurement information proposed in this paper can be concluded as follows: 


\section{A. Equivalent generator and load model}

generator model takes frequency as the input signal shown in type (1), electromagnetic power and load shown in type (2) and type (3).

$$
\begin{aligned}
& T_{j} \frac{d \omega}{d t}=P_{m}-P_{e}-D \frac{d \delta}{d t} \\
& \frac{d \delta}{d t}=\left(\omega-\omega_{f}\right) \omega_{0} \\
& T_{d}^{\prime} \frac{d E^{\prime}}{d t}=E_{f 0}-K_{V}\left(V-V_{0}\right)-\frac{x_{d}}{x_{d}^{\prime}} E^{\prime}+\left(\frac{x_{d}}{x_{d}^{\prime}}-1\right) V \cos (\delta) \\
& P_{e}=\frac{E^{\prime} V}{x_{d}^{\prime}} \sin (\delta) \\
& Q_{e}=\frac{E^{\prime} V \cos (\delta)-V^{2}}{x_{d}^{\prime}} \\
& P_{s}=P_{s 0}\left(V / V_{0}\right)^{2} \\
& Q_{s}=Q_{s 0}\left(V / V_{0}\right)^{2}
\end{aligned}
$$

The tie line power equation is:

$$
\left\{\begin{array}{l}
P_{l}=P_{e}-P_{s} \\
Q_{l}=Q_{e}-Q_{s}
\end{array}\right.
$$

Where, $T_{j}$ is inertia time constant; $P_{m}$ is mechanical power; $E$ is potential back of $x_{d}^{\prime}$; $\mathrm{V}$ is generator voltage, $\mathrm{V} 0$ is the initial voltage of the machine; $x_{d}$ is transient reactance of $\mathrm{d}$ axis, $x_{d}$ is synchronous reactance of $\mathrm{d}$ axis; $\delta$ is the angle between the voltage and the electric potential; $D$ is damping coefficient; $\omega$ is rotor speed, $\omega_{f}$ is bus frequency; $T_{d 0}$ is $\mathrm{d}$ axis transient open circuit time constant; $E_{f 0}$ is initial excitation voltage; $K_{v}$ is the excitation voltage feedback coefficient; $P_{s 0} 、 Q_{s 0}$ is load base value of active and reactive power respectively; $P_{l}, Q_{l}$ is active and reactive power on tie-line respectively. Type (1) - (4) constitute dynamic equivalence model of small hydropower group.

\section{B. Equivalent parameter identification}

Four input variables of equivalent model are the actual voltage, frequency, power of tie-line respectively $\left[V_{\text {real }} 、 \omega_{\text {real }} 、 P_{\text {lreal }} 、 Q_{\text {lreal }}\right]$, output variables are active power and reactive power identified $\left[P_{l}, Q_{l}\right]$, eight parameters to be identified are $\left[x_{d}, x_{d}, T_{d 0}, T_{j}, D 、 K_{v} 、 P_{s 0} 、 Q_{s 0}\right]$.

Injecting the voltage, frequency, power signal of tie-line after disturbance occurs in external power grid into the equivalent model, which will produce equivalent active power and reactive power of tie-line $P_{l} 、 Q_{l}$. Combined with the tie line power oscillation waveform, deviation function defined as:

$$
\left\{\begin{array}{l}
e=\sum_{i=1}^{i \leq n} \sqrt{A(i)^{2}+B(i)^{2}} \\
A(i)=P_{l}(i)-P_{\text {lreal }}(i) \\
B(i)=Q_{l}(i)-Q_{\text {lreal }}(i)
\end{array}\right.
$$

Where, $n$ is sampling points of oscillation waveform data. Using the improved particle swarm optimization (pso) algorithm to search parameters, when the deviation convergence to minimum, equivalent system parameters is identified.

\section{Disturbance Data Selection}

For the Equivalent accuracy of hydropower generator group in a particular area, it's associated with the disturbance intensity and the disturbance source location. But the there is no uniform standard about electric distance of the fault and equivalent area and the size of the fault, and it's difficult to measure. 
Partition scheme according to the hydropower generator group's geographical position shown in figure 1. Region $1 \sim 5$ represent hydropower bases; region $6 \sim 10$ represent the main power grid. For an example, equivalent for hydropower generator group in regional 5 . When disturbance occurs near regional 6, small disturbance can fully inspire the dynamic characteristics of the generator group to be equivalent; When the disturbance occurs in area 10, need stronger disturbance; For the disturbance in area 8 , it not necessarily inspire the real characteristics.

In order to select disturbance data for dynamic equivalent more reasonable, a index use tie-line power oscillation to evaluate disturbance intensity is proposed, it defined as follows:

$\left\{\begin{array}{l}f_{s}=\frac{\Delta P_{\max i j}}{P_{s t i j}} \times 100 \% \\ f_{s}>\varepsilon_{i j}\end{array}\right.$

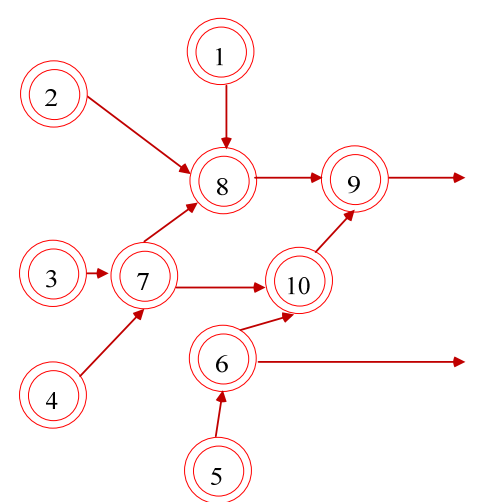

Fig.1. The power grid partition map

Where, $f_{s}$ is index to evaluate disturbance intensity; $\Delta P_{\text {maxij }}$ is tie-line maximum power fluctuations value in $\mathrm{j}$-th area when fault occurs in $\mathrm{i}$-th region; $P_{s t}$ is the steady state power of tie-line; $\varepsilon_{i j}$ is the minimum index value to evaluate disturbance intensity that able to accurately identify the equivalent parameters of generator group in $\mathrm{j}$-th area when fault occurs in $\mathrm{i}$-th region. Therefore, according to the topology of power grid, evaluate the minimum index value $\varepsilon_{i j}$ of each area, select disturbance data based on this method can get more accurate equivalent parameters.

\section{Application in the actual system}

\section{A. Disturbance data selection}

According to the equal area low ${ }^{[5]}$, the system transient stability is strongly influenced by $T_{j}$, $x_{d}{ }^{\prime}$. According to damping ratio and undamped natural frequency of single machine infinite system described with classical generator model ${ }^{[11]}$, increase the damping torque coefficient $D$ will increase the damping ratio, while the increase of the inertial time constant will make the undamped natural frequency and damping ratio decreases.

Because of the equivalent model contains load, it's difficult to directly compare the damping ratio between the actual generator and equivalent generator. Reference 6 pointed out that $T_{j} 、 x_{d}, P_{s 0}$ to the equivalent error sensitivity is higher, the rest of the parameter sensitivity is low. Therefore, the influence of disturbance intensity on equivalent accuracy can indirectly represented as the influence to the accuracy of equivalent inertia time constant, transient reactance and load value.

Take a generator known parameters $\left(P_{s 0}=0 ; P_{n}=550 \mathrm{MW} ; T_{j}=53.613 \mathrm{~s}, x_{d}{ }^{\prime}=0.0523 p \cdot u\right.$ based on 100 MVA) of area 5 in figure 1 for example. The equivalent inertia time constant and transient reactance identification error with $f_{s}$ change trend under the disturbances nearby (area 6) and in the distance (area 8) shown in figure 2.

Considering the above analysis, disturbance nearby the area to be equivalent, equivalent is accurate when $f_{s}>15 \%, T_{j}, x_{d}{ }^{\prime}$ error $<5 \%$; disturbance in the distance, can not necessarily make identification parameters accurate. The reason is that the distant fault is not necessarily inspire the real characteristics of system to be equivalent. So, disturbance data selection can refer to the following principles: (a) disturbance occurs outside the region to be equivalent, and close to the 
equivalent area; (b) $\varepsilon_{i j}$ value can be $15 \%$, if select fault in the distance, disturbance intensity should strong enough, $\varepsilon_{i j}$ can be $60 \%$, but every $\varepsilon_{i j}$ should be evaluated respectively.

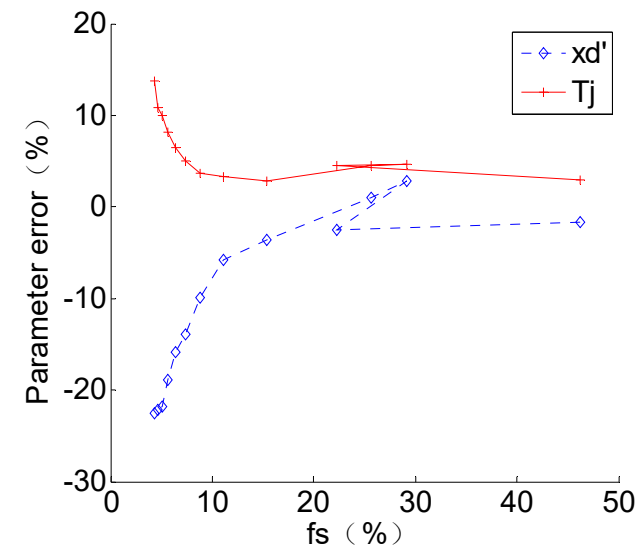

(a) The fault nearby equivalent

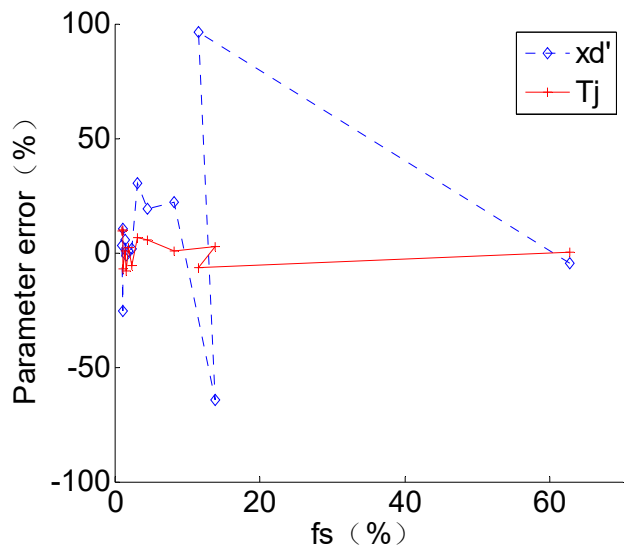

(b) The fault in distance

Fig.2. Error of equivalent inertial time constant and transient reactance along with $f_{s}$

\section{B. PMU measured disturbance data identification}

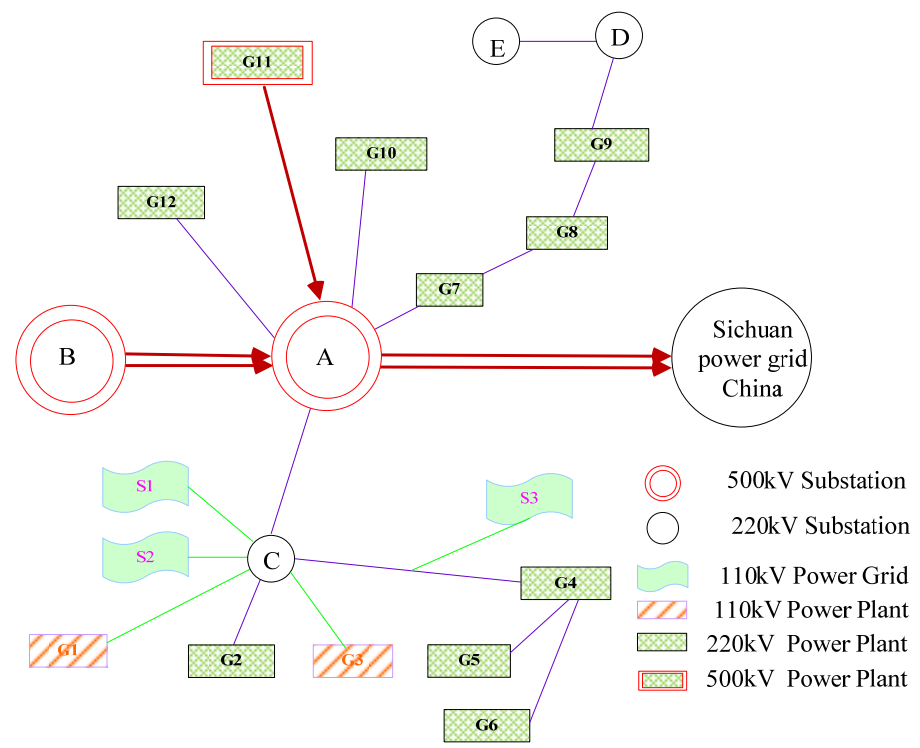

Fig.3. A hydropower channel of Sichuan power grid

Using the southern Sichuan hydropower channel as equivalent verification model shown in figure 3. Large scale hydropower and some small $110 \mathrm{kV}$ power grid link to Sichuan power grid by line AC, besides there are some small hydropower generators and local power network. In the actual mechanical and electrical transient calculations, $110 \mathrm{kV}$ generators and power grids link to substation $\mathrm{C}$ are not considered in detail, It's instead of negative constant power load and a part of generators, which will seriously affect the accuracy of the simulation.

According to the disturbance selection method, use the PMU data $\left(\varepsilon_{i j}=20 \%\right)$ of line ac when fault occurred in Sichuan power grid at 0:13 on November 6, 2015. The grid connect to substation $\mathrm{C}$ will be equivalent. Power response curve comparison before and after the equivalent shown in figure 4, oscillation mode comparison before and after the equivalent shown in table 1 . Curve marked as a negative load is response that system link to substation $\mathrm{C}$ equivalent as negative constant power load and a part of generators, curve marked as equivalent system is response that system link to substation $\mathrm{C}$ equivalent by PMU data. 


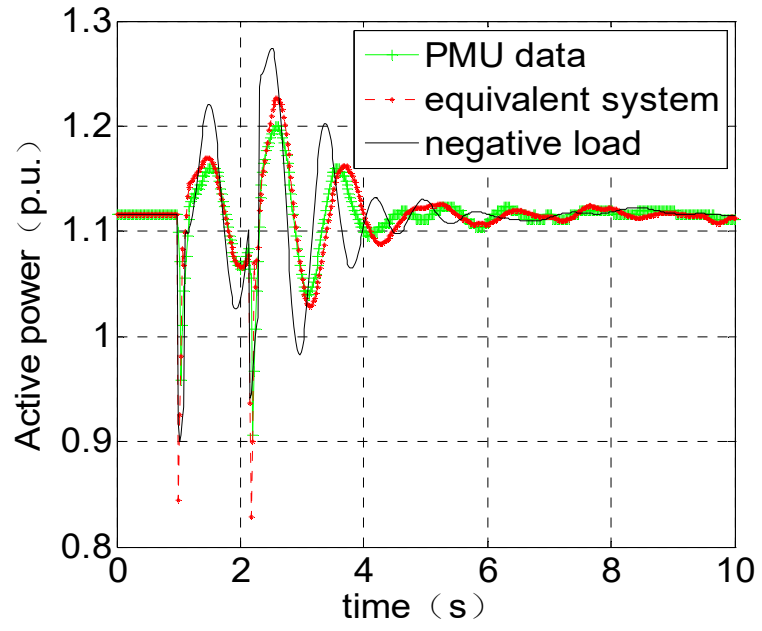

(a) Active power

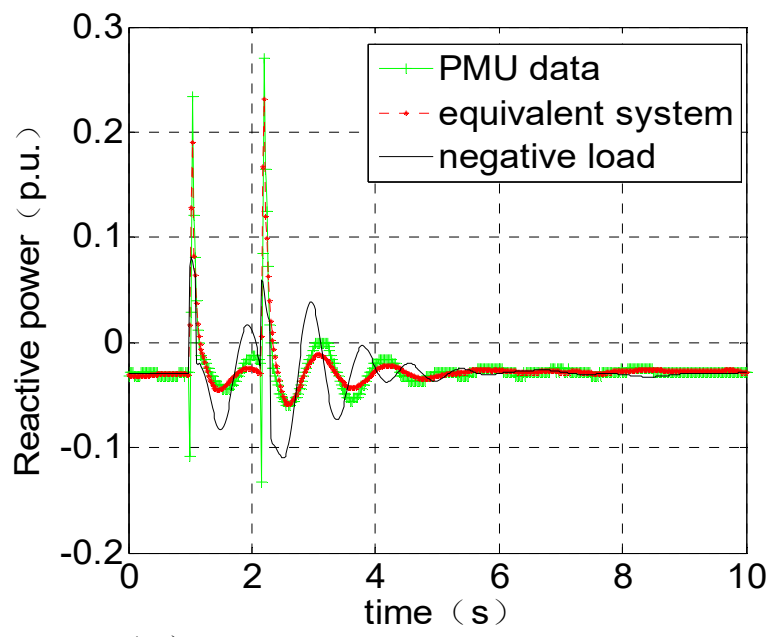

(b) Reactive power

Fig.4. Power response curve comparison before and after the equivalent

Table 1 Oscillation mode comparison before and after the equivalent

\begin{tabular}{|c|c|c|c|}
\hline Data type & mode & frequency $(\mathrm{Hz})$ & damping ratio $(\%)$ \\
\hline \multirow{2}{*}{ original response record by PMU } & 1 & 0.96 & 15.01 \\
\hline & 2 & 0.78 & 13.01 \\
\hline \multirow{2}{*}{ equivalent by PMU data } & 1 & 0.94 & 17.75 \\
\hline & 2 & 0.77 & 11.86 \\
\hline \multirow[t]{2}{*}{ negative load } & 1 & 1.28 & 15.73 \\
\hline & 2 & 0.83 & 29.12 \\
\hline
\end{tabular}

Calculated by the above, oscillation waveform of the equivalent system is similar to the original response record by PMU, the main oscillation mode is similar too. The response of system described by negative constant power load and a part of generators is different with the original curve.

\section{Software development and practical application}

Based on the dynamic equivalence method put forward, dynamic equivalent software for hydropower generator group has been developed. It's working process is as follows:

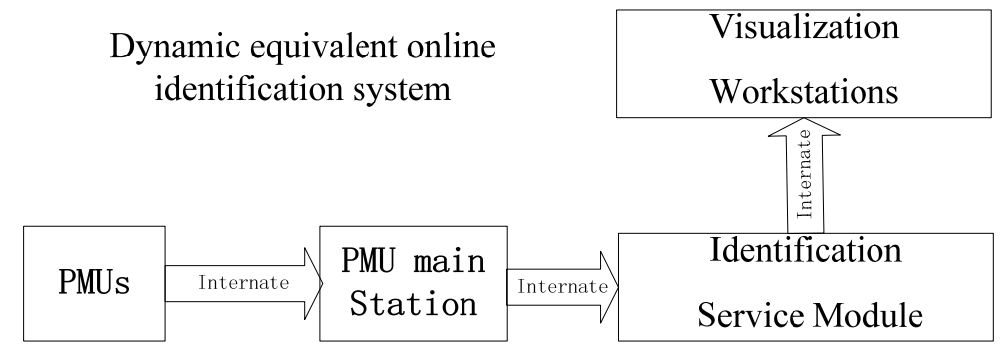

Fig.5. Structure of the dynamic equivalent online identification system 


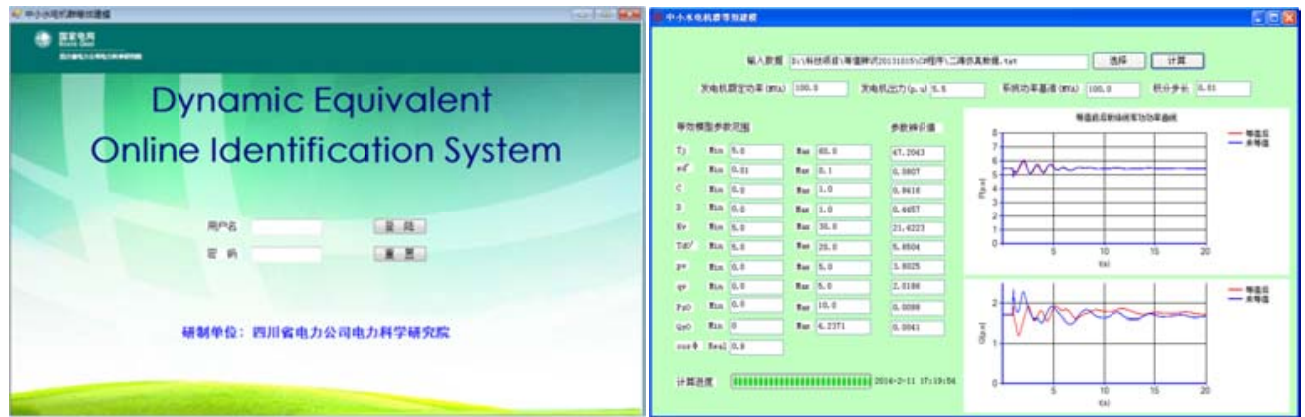

Fig.6. Interface of the dynamic equivalent online identification system

\section{Conclusion}

The application effect of dynamic equivalent method for hydropower generator group based on phasor measurement information and disturbance data selection method in actual power grid is good. The successful application of the software system proved the correctness and effectiveness of the equivalent method.

\section{References}

[1] JU Ping, WANG Wei-hua, XIE Hong-jie, etc. Identification approach to dynamic equivalents of the power system interconnected with three areas[J]. Proceedings of the CSEE, 2007, 27(13): 29-34.

[2] WANG Min, WEN Jin-yu, HU Wen-bo, et al. A dynamic equivalent modeling for regional small hydropower generator group[J]. Power System Protection and Control, 2013, 41(17): 1-9.

[3] LI Xiao-hui, LUO Min, LIU Li-xia, et al. A new dynamic equivallence method and its application in Tianjin Grid[J]. Power System Protection and Control, 2010, 38(3): 61-66.

[4] SHI Huabo,CHEN Gang,DING Lijie, ZHANG Hua. Research on Dynamic Equivalent Method for Hydropower Generator Group Based on Phasor Measurement Information Part I :Theory and Method, 2016 4th International Conference on Energy, Power and Environmental Engineering (ICEPEE 2016), Tianjin, China.

[5] PRABHA KUNDUR,Power System Stability and Control[M]. Bejing : China Electric Power Press,2001.

[6] HU Bowei,SUN Jingtao,DING Lijie. Dynamic Equivalent Modeling for Small and Medium Hydropower Generator Group based on measurements[J].energies,2016(Has been submitted). 